\title{
Article \\ The Strong Equitable Vertex 1-Arboricity of Complete Bipartite Graphs and Balanced Complete $k$-Partite Graphs
}

\author{
Janejira Laomala ${ }^{1}$, Keaitsuda Maneeruk Nakprasit ${ }^{1}$, Kittikorn Nakprasit $^{1}$ and Watcharintorn Ruksasakchai ${ }^{2, *}$ (D) \\ 1 Department of Mathematics, Faculty of Science, Khon Kaen University, Khon Kaen 40002, Thailand; \\ janejira.1@kkumail.com (J.L.); keaina@kku.ac.th (K.M.N.); kitnak@kku.ac.th (K.N.) \\ 2 Department of Mathematics, Statistics and Computer Science, Faculty of Liberal Arts and Science, \\ Kasetsart University, Kamphaeng Saen Campus, Nakhon Pathom 73140, Thailand \\ * Correspondence: faaswtr@ku.ac.th
}

Citation: Laomala, J.; Nakprasit, K.M.; Nakprasit, K.; Ruksasakchai, W. The Strong Equitable Vertex 1-Arboricity of Complete Bipartite Graphs and Balanced Complete k-Partite Graphs. Symmetry 2022, 14, 287. https://doi.org/10.3390/ sym 14020287

Academic Editor: Domenico Labbate

Received: 7 January 2022

Accepted: 26 January 2022

Published: 31 January 2022

Publisher's Note: MDPI stays neutral with regard to jurisdictional claims in published maps and institutional affiliations.

Copyright: (c) 2022 by the authors. Licensee MDPI, Basel, Switzerland. This article is an open access article distributed under the terms and conditions of the Creative Commons Attribution (CC BY) license (https:// creativecommons.org/licenses/by/ $4.0 /)$.

\begin{abstract}
An equitable $k$-coloring of a graph $G$ is a proper $k$-coloring of $G$ such that the sizes of any two color classes differ by at most one. An equitable $(q, r)$-tree-coloring of a graph $G$ is an equitable $q$-coloring of $G$ such that the subgraph induced by each color class is a forest of maximum degree at most $r$. Let the strong equitable vertex $r$-arboricity of a graph $G$, denoted by $v a_{r} \equiv(G)$, be the minimum $p$ such that $G$ has an equitable $(q, r)$-tree-coloring for every $q \geq p$. The values of $v a_{1}^{\equiv}\left(K_{n, n}\right)$ were investigated by Tao and Lin and $\mathrm{Wu}, \mathrm{Zhang}$, and $\mathrm{Li}$ where exact values of $v a_{1} \equiv\left(K_{n, n}\right)$ were found in some special cases. In this paper, we extend their results by giving the exact values of $v a_{1}\left(K_{n, n}\right)$ for all cases. In the process, we introduce a new function related to an equitable coloring and obtain a more general result by determining the exact value of each $v a_{1} \equiv\left(K_{m, n}\right)$ and $v a_{1}^{\equiv}(G)$ where $G$ is a balanced complete $k$-partite graph $K_{n, \ldots, n}$. Both complete bipartite graphs $K_{m, n}$ and balanced complete $k$-partite graphs $K_{n, \ldots, n}$ are symmetry in several aspects and also studied broadly. For the other aspect of symmetry, by the definition of equitable $k$-coloring of graphs, in a specific case that the number of colors divides the number of vertices of graph, we can say that the graph is a balanced $k$-partite graph.
\end{abstract}

Keywords: equitable coloring; $k$-tree-coloring; vertex $k$-arboricity; complete multipartite graph

\section{Introduction}

Throughout this paper, all graphs considered are finite and simple. Let $V(G)$ and $E(G)$ denote the vertex set and edge set of a graph $G$ respectively. Let $K_{n_{1}, \ldots, n_{k}}$ be a complete $k$-partite graph in which partite set $X_{i}$ has size $n_{i}$ for $1 \leq i \leq k$. Let $K_{k * n}$ denote a complete $k$-partite graph with each partite set has size $n$, and naturally we always assume that $k \geq 2$. For a real number $r,\lfloor r\rfloor$ is the largest integer number not greater than $r$, and $\lceil r\rceil$ is the smallest integer number not less than $r$. A $k$-set is a set with $k$ elements.

An equitable $k$-coloring of a graph is a proper vertex $k$-coloring such that the sizes of every two color classes differ by at most 1 . A graph $G$ is equitably $k$-colorable if $G$ admits an equitable $k$-coloring, equivalently if $V(G)$ can be partitioned into $k$ independent sets where each set is a $\lfloor|V(G)| / k\rfloor$-set or a $\lceil|V(G)| / k\rceil$-set. The equitable chromatic number of a graph $G$ is the minimum number $k$ such that $G$ is equitably $k$-colorable. In contrast with ordinary proper coloring, a graph may have an equitable $k$-coloring but has no equitable $(k+1)$-coloring. For example, $K_{7,7}$ has an equitable $k$-coloring for $k=2,4,6$ and $k \geq 8$, but has no equitable $k$-coloring for $k=3,5$, and 7 . This leads to the definition of the equitable chromatic threshold which is the minimum $p$ such that $G$ is equitably $q$-colorable for every $q \geq p$.

The topic of equitable coloring, introduced by Meyer [1], was motivated by a problem of municipal garbage collection [2]. To model the problem, the vertices of the graph are used to represent garbage collection routes. If two routes cannot be run in the same day, then two corresponding vertices share the same edge. Thus the problem of assigning routes for $k$ days satisfying the number of routes run on each day are about the same can be represented 
by an equitable $k$-coloring. A similar approach can be applied to scheduling [3-6] and modeling load balance in parallel memory systems $[7,8]$.

In [9], Fan et al introduced an equitable relaxed coloring in which every color class induces a forest with maximum degree at most 1 and the sizes of any two color classes differ by at most 1 . On the basis of the aforementioned research, Wu, Zhang, and Li [10] introduced an equitable $(q, r)$-tree-coloring of a graph $G$ which is a $q$-coloring of vertices of $G$ such that the subgraph induced by each color class is a forest of maximum degree at most $r$ and the sizes of any two color classes differ by at most one. In other words, a graph $G$ has an equitable $(q, r)$-tree-coloring if $V(G)$ can be partitioned into $q$ sets such that each set is a $\lfloor|V(G)| / q\rfloor$ - or $\lceil|V(G)| / q\rceil$-set inducing a forest with maximum degree at most $r$. Let the strong equitable vertex $k$-arboricity, denoted by $v a_{r} \equiv(G)$, be the minimum $p$ such that $G$ has an equitable $(q, r)$-tree-coloring for every $q \geq p$. The values of $v a_{1} \equiv\left(K_{n, n}\right)$ were investigated by Tao and Lin [11] and $\mathrm{Wu}$, Zhang, and Li [10] where exact values of $v a_{1}\left(K_{n, n}\right)$ were found in some special cases. In this paper, we extend their results by giving the exact values of $v a_{1}\left(K_{n, n}\right)$ for all cases. In the process, we introduce a new function related to an equitable coloring and furthermore, obtain a general result by determining the exact value of each $v a_{1} \equiv\left(K_{m, n}\right)$ and $v a_{1} \equiv(G)$ where $G$ is a balanced complete $k$-partite graph $K_{n, \ldots, n}$.

\section{Helpful Lemmas}

To find $v a_{1} \equiv\left(K_{m, n}\right)$ and $v a_{1} \equiv\left(K_{k * n}\right)$, we introduce the notion of $p\left(q: n_{1}, \ldots, n_{k}\right)$ which can be computed in linear time.

Definition 1. Assume that $K_{n_{1}, \ldots, n_{k}}$ has an equitable $q$-coloring. Define $p\left(q: n_{1}, \ldots, n_{k}\right)=$ $\left\lceil n_{1} / d\right\rceil+\cdots+\left\lceil n_{k} / d\right\rceil$ where $d$ is the minimum integer not less than $\left\lceil\left(n_{1}+\cdots+n_{k}\right) / q\right\rceil$ satisfying at least one of the following conditions:

(i) there exist $n_{i}$ and $n_{j}(i \neq j)$ not divisible by $d$;

(ii) there exists $n_{i}$ with $n_{i} /\left\lfloor n_{i} / d\right\rfloor>d+1$.

Lemma 1. Assume $G=K_{n_{1}, \ldots, n_{k}}$ has an equitable $q$-coloring. If $p\left(q: n_{1}, \ldots, n_{k}\right) \leq r \leq q$, then $G$ has an equitable r-coloring.

Proof. Let $p=p\left(q: n_{1}, \ldots, n_{k}\right)$ and $N=n_{1}+\cdots+n_{k}$. We prove by reverse induction that $G$ has an equitable $r$-coloring when $p \leq r \leq q$. By assumption, $G$ has an equitable $q$-coloring. Assume $G$ has an equitable $r$-coloring where $p<r \leq q$. Let $b=\lceil N / r\rceil$. It follows that, for $1 \leq i \leq k$, there are non-negative integers $r_{i}$ and $s_{i}$ where $r_{i}-s_{i} \geq 0$ such that $n_{i}=\left(r_{i}-s_{i}\right) b+s_{i}(b-1)=r_{i} b-s_{i}$ and $r=r_{1}+\cdots+r_{k}$.

Case 1: There exists $j$ with $r_{j} \neq\left\lceil n_{j} / b\right\rceil$.

Note that $n_{j}=\left\lceil n_{j} / b\right\rceil b-g_{j}$ for some $g_{j}$ satisfying $0 \leq g_{j} \leq b-1$. From $r_{j} b-s_{j}=$ $\left\lceil n_{j} / b\right\rceil b-g_{j}$, we obtain $\left(r_{j}-\left\lceil n_{j} / b\right\rceil\right) b=s_{j}-g_{j}$. Since $r_{j} \neq\left\lceil n_{j} / b\right\rceil, 0 \leq g_{j} \leq b-1$, and $s_{j}$ is non-negative, it follows that $s_{j}-g_{j}$ is a positive multiple of $b$. Rewrite $n_{j}=$ $\left(r_{j}-s_{j}\right) b+s_{j}(b-1)$ into $n_{j}=\left(r_{j}-s_{j}+b-1\right) b+\left(s_{j}-b\right)(b-1)$. Since $s_{j}-g_{j}$ is a positive multiple of $b$ and $g_{j}$ is non-negative, it follows that $s_{j}-b$ is non-negative. Thus we can partition $X_{j}$ into $r_{j}-s_{j}+b-1$ color classes of size $b$ and $s_{j}-b$ color classes of size $b-1$. That is, we can partition $X_{j}$ into $r_{j}-1$ sets of size $b$ or $b-1$. Since we can partition all other $X_{i}$ into $r_{i}$ color classes of size $b$ or $b-1$ and $\left(\sum_{i \neq j} r_{i}\right)+\left(r_{j}-1\right)=\left(\sum_{i=1}^{k} r_{i}\right)-1=r-1$, the graph $G$ has an equitable $(r-1)$-coloring.

Case 2: $r_{i}=\left\lceil n_{i} / b\right\rceil$ for $1 \leq i \leq k$.

In this case, $r=\left\lceil n_{1} / b\right\rceil+\cdots+\left\lceil n_{k} / b\right\rceil$. Since $r>p=\left\lceil n_{1} / d\right\rceil+\cdots+\left\lceil n_{k} / d\right\rceil$, it follows that $d>b$. Definition 1 implies that $b$ satisfies neither conditions (i) nor (ii) in the definition. Thus we may assume $n_{i}=r_{i} b$ for $2 \leq i \leq k$, and $n_{j} /\left\lfloor n_{j} / b\right\rfloor \leq b+1$ for $1 \leq j \leq k$.

Subcase 2.1: $n_{1} \neq r_{1} b$.

Then $b<n_{1} /\left\lfloor n_{1} / b\right\rfloor=n_{1} /\left(\left\lceil n_{1} / b\right\rceil-1\right)=n_{1} /\left(r_{1}-1\right)$. Since $b$ violates condition (ii), we have $n_{1} /\left(r_{1}-1\right)=n_{1} /\left\lfloor n_{1} / b\right\rfloor \leq b+1$. Thus $b<n_{1} /\left(r_{1}-1\right) \leq b+1$. Consequently, 
we can partition $n_{1}$ into $r_{1}-1$ color classes of size $b$ or $b+1$. Combining with $r_{i}$ color classes of $X_{i}$ of size $b$ for $i \geq 2$, we have an equitable $(r-1)$-coloring.

Subcase 2.2: $n_{1}=r_{1} b$.

It follows that $n_{i}=r_{i} b$ for $1 \leq i \leq k$. If there is $j$ such that $n_{j} /\left(r_{j}-1\right) \leq b+1$, then we have an equitable $(r-1)$-coloring as in subcase 2.1. Thus we may assume that $n_{i} /\left(r_{i}-1\right)>b+1$ for $1 \leq i \leq k$.

We claim that $b+1 \geq d$ and $r_{i}=\left\lceil n_{i} / b\right\rceil=\left\lceil n_{i} /(b+1)\right\rceil$. If the claim holds, we have $r=\sum_{i=1}^{k}\left\lceil n_{i} / b\right\rceil \leq \sum_{i=1}^{k}\left\lceil n_{i} / d\right\rceil=p$ which contradicts the fact that $r>p$. Thus this situation is impossible. To prove the claim, first we suppose to the contrary that there is $n_{i}$ which is divisible by $b+1$. Since $n_{i}=r_{i} b$, we have $r_{i}=t_{i}(b+1)$ for some positive integer $t_{i}$. Consequenly, $n_{i} /\left(r_{i}-1\right)=t_{i}(b+1) b /\left(t_{i}(b+1)-1\right)=b+b /\left(t_{i}(b+1)-1\right) \leq b+1$ which contradicts the fact that $n_{i} /\left(r_{i}-1\right)>b+1$. Thus $n_{i}$ is not divisible by $b+1$ where $1 \leq i \leq k$. By condition (i) in Definition 1 , we have $b+1 \geq d$. Since $n_{i}=r_{i} b$ and $n_{i} /\left(r_{i}-1\right)>b+1$ where $1 \leq i \leq k$, it follows that $r_{i}=n_{i} / b>n_{i} /(b+1)>r_{i}-1$. This leads to $r_{i}=\left\lceil n_{i} / b\right\rceil=\left\lceil n_{i} /(b+1)\right\rceil$. Thus, we have the claim and this completes the proof.

Example 1. Let $G=K_{1,1,2,2,3}$. Then $G$ has an equitable 8-coloring. One can compute that $p=p\left(q: n_{1}, \ldots, n_{k}\right)=6$. We can notice that $G$ has an equitable $r$-coloring for every $6 \leq r \leq 8$ as in Lemma 1 .

Lemma 2. If $G=K_{n_{1}, \ldots, n_{k}}$ has an equitable q-coloring, then $G$ has no equitable $(p-1)$-coloring where $p=p\left(q: n_{1}, \ldots, n_{k}\right)$.

Proof. Recall that $p=\left\lceil n_{1} / d\right\rceil+\cdots+\left\lceil n_{k} / d\right\rceil$ where $d$ is as in Definition 1 . Suppose to the contrary that $G$ has an equitable $(p-1)$-coloring. Then there is a partite set, say $X_{1}$ of size $n_{1}$, partitioned into at most $\left\lceil n_{1} / d\right\rceil-1$ color classes. Consequently, there is a color class containing vertices in $X_{1}$ with size at least $d+1$. If there is $X_{j}$ partitioned into at least $\left\lceil n_{j} / d\right\rceil+1$ color classes, then there is a color class containing vertices in $X_{j}$ with size at most $d-1$. So we have two color classes with sizes differed by at least 2 , a contradiction. Thus we assume that $X_{1}$ is partitioned into $\left\lceil n_{1} / d\right\rceil-1$ color classes, and $X_{j}$ is partitioned into $\left\lceil n_{j} / d\right\rceil$ color classes for each $j \in\{2, \ldots, k\}$. We consider two cases.

Case 1: There exists $j \in\{2, \ldots, k\}$ such that $n_{j}$ is not divisible by $d$.

It follows that there is a color class containing vertices in $X_{j}$ with size at most $d-1$. So we have two color classes with sizes differed by at least 2, a contradiction.

Case $2: X_{j}$ is partitioned into exactly $\left\lceil n_{j} / d\right\rceil$ color classes and $n_{j}$ is divisible by $d$ for each $j \in\{2, \ldots, k\}$.

Recall that $d$ is as in Definition 1. It follows that $n_{1}$ has $n_{1} /\left\lfloor n_{1} / d\right\rfloor>d+1$. Since $X_{1}$ is partitioned into $\left\lceil n_{1} / d\right\rceil-1$ color classes, there is a color class containing vertices in $X_{1}$ with size at least $d+2$. However, each color class containing vertices in $X_{j}(j \in\{2, \ldots, k\})$ has size $d$, so we have a contradiction. Thus $G$ has no equitable $(p-1)$-coloring.

Example 2. Let $G=K_{2,3,4}$. Then $G$ has an equitable 4-coloring. One can compute that $p=p\left(q: n_{1}, \ldots, n_{k}\right)=4$. We can notice that $G$ cannot have an equitable 3 -coloring as in Lemma 2.

Lemmas 1 and 2 yield the following theorem. Note that $K_{n_{1}, \ldots, n_{k}}$ has an equitable $r$-coloring when $r \geq n_{1}+\cdots+n_{k}$.

Theorem 1. If $K_{n_{1}, \ldots, n_{k}}$ has an equitable q-coloring, then $p\left(q: n_{1}, \ldots, n_{k}\right)$ is the minimum $p$ such that $G$ has an equitable $r$-coloring for each $r$ satisfying $p \leq r \leq q$. In particular, the equitable chromatic threshold of $K_{n_{1}, \ldots, n_{k}}$ is equal to $p\left(n_{1}+\cdots+n_{k}: n_{1}, \ldots, n_{k}\right)$.

We use $p\left(q: n_{1}, \ldots, n_{k}\right)$ to find $v a_{1} \equiv\left(K_{n_{1}, \ldots, n_{k}}\right)$ as follows. 
Lemma 3. Let $G=K_{n_{1}, \ldots, n_{k}}$ and $N=n_{1}+\cdots+n_{k}$. If $G$ has an equitable q-coloring where $N /(q-1) \geq 3$ and $G$ has an equitable $(r, 1)$-tree-coloring for each $r \geq q$, then $v a \equiv$ $\left.n_{1}, \ldots, n_{k}\right)$.

Proof. Let $p=p\left(q: n_{1}, \ldots, n_{k}\right)$. From the assumption for $q$ and Definition 1, the graph $G$ has an equitable $(r, 1)$-tree coloring for each $r \geq p$. It remains to show that $G$ has no equitable $(p-1,1)$-tree-coloring. Suppose to the contrary that $G$ has an equitable $(p-1,1)$ tree-coloring. Since $p-1 \leq q-1$, there is a color class of size at least $N /(p-1) \geq$ $N /(q-1) \geq 3$ that is not independent. Observe that the graph induced by this color class has maximum degree greater than 1 , which is a contradiction. This completes the proof.

\section{The Strong Equitable Vertex 1-Arboricity of $K_{m, n}$}

Let two partite sets of $K_{m, n}$ be $X_{1}$ and $X_{2}$ where $X_{1}=\left\{u_{1}, \ldots, u_{m}\right\}$ and $X_{2}=$ $\left\{v_{1}, \ldots, v_{n}\right\}$.

Lemma 4. If $m=3 b+g$ and $n=3 c+h$ where $b$ and $c$ are non-negative integers and $0 \leq g, h \leq$ 2 , then $v a_{1} \equiv\left(K_{m, n}\right) \leq b+c+2$.

Proof. Let $N=m+n$. First, consider $q \geq N / 2$. We partition $V\left(K_{m, n}\right)$ into $q$ sets equitably. Each resulting set has size not greater than 2 , so it cannot induce a graph with maximum degree more than 1 . Thus we have an equitable $(q, 1)$-tree-coloring.

Now assume $b+c+2 \leq q<N / 2$. It follows that $2<N / q<3$. Thus there are positive integers $r$ and $s$ such that $N=3 r+2 s$ and $q=r+s$. Since $q \geq b+c+2$, we have $r \leq b+c$.

Let $X_{1}^{\prime}$ be a (3b)-subset of $X_{1}$ and let $X_{2}^{\prime}$ be a (3c)-subset of $X_{2}$ (One of these sets may be empty.) Partition $X_{1}^{\prime}$ into $b 3$-sets and partition $X_{2}^{\prime}$ into $c 3$-sets. Since $r \leq b+c$, we choose $r$-sets from these $b+c$ sets to initiate a new partition. Next, we partition set of the remaining $N-3 r$ vertices into s 2-sets. Since each of the resulting 3-sets contains vertices from the same partite set, it is an independent set. Moreover, each of remaining 2 -sets cannot induce a graph with maximum degree more than 1 . Thus this partition is an equitable $(q, 1)$-tree-coloring for each $q \geq b+c+2$.

Lemma 5. For any positive integer $n, v a_{1} \equiv\left(K_{1, n}\right)=\lceil(n+2) / 3\rceil$.

Proof. Let $r=\lceil(n+2) / 3\rceil$, and let $n=3 b+h$ for some integer $h$ where $0 \leq h \leq 2$. Observe that $r=b+1$ when $h=0$ or 1 , and $r=b+2$ when $h=2$. First, we show that $v a_{1}\left(K_{1, n}\right) \leq r$. From Lemma 4 , we know that $K_{1, n}$ has an equitable $(q, 1)$-tree-coloring for each $q \geq b+2$. It remains to show that $K_{1, n}$ has an equitable $(b+1,1)$-tree-coloring if $h=0$ or 1 .

From $2 \leq(n+1) /(b+1)=(3 b+h+1) /(b+1)<3$, there are a positive integer $r$ and a non-negative integer $s$ such that $n+1=3 r+2 s$ and $r+s=b+1$. Choosing $u_{1}$ and another vertex in $X_{2}$ to be in one set, and partitioning the set of remaining vertices into $b$ sets equitably, we obtain an equitable $(b+1,1)$-tree-coloring.

Next, we show that $K_{1, n}$ has no equitable $(r-1,1)$-tree-coloring to complete the proof. Suppose to the contrary that $K_{1, n}$ has an equitable $(r-1,1)$-tree-coloring. However, $(n+1) /(r-1) \geq 3$. Consequently, every resulting color class has size at least 3 . Then a color class containing $u_{1}$ induces a graph with maximum degree greater than 1 , a contradiction. This completes the proof.

Lemma 6. For any positive integer $n, v a_{1} \equiv\left(K_{2, n}\right)=\lceil(n+3) / 3\rceil$.

Proof. Let $r=\lceil(n+3) / 3\rceil$, and let $n=3 b+h$ for some integer $h$ with $0 \leq h \leq 2$. Observe that $r=b+1$ when $h=0$, and $r=b+2$ when $h=1$, or 2. First, we show that $v a_{1} \equiv\left(K_{2, n}\right) \leq r$. From Lemma 4 , we know that $K_{2, n}$ has an equitable $(q, 1)$-tree-coloring 
for each $q \geq b+2$. It remains to show that $K_{2, n}$ has an equitable $(b+1,1)$-tree-coloring if $n=3 b$.

Since $2 \leq(n+2) /(b+1)=(3 b+2) /(b+1)<3$, there are a positive integer $r$ and a non-negative integer $s$ such that $n+2=3 r+2 s$ and $r+s=b+1$. Choosing $u_{1}$ and $u_{2}$ to be in one set, and partitioning the set of remaining vertices into $b$ sets equitably, we obtain an equitable $(b+1,1)$-tree-coloring.

Next, we show that $K_{2, n}$ has no equitable $(r-1,1)$-tree-coloring to complete the proof. Suppose to the contrary that $K_{2, n}$ has an equitable $(r-1,1)$-tree-coloring. Since $(2+n) /(r-1) \geq 3$, every color class has size at least 3 . Thus a color class containing $u_{1}$ induces a graph with maximum degree greater than 1 , a contradiction.

Lemma 7. If $m=3 b$ and $n=3 c$ for some positive integers $b$ and $c$, then $v a \equiv\left(K_{m, n}\right)=p(b+c$ : $m, n)$.

Proof. From Lemma 4, the graph $K_{m, n}$ has an equitable $(q, 1)$-tree-coloring for each integer $q$ such that $q \geq b+c+2$.

Next, we show that $K_{m, n}$ has an equitable $(b+c+1,1)$-tree-coloring. We initiate a partition by assigning three 2-sets $\left\{u_{1}, v_{1}\right\},\left\{u_{2}, v_{2}\right\},\left\{u_{3}, v_{3}\right\}$ and then partition $X_{1}-$ $\left\{u_{1}, u_{2}, u_{3}\right\}$ into $b-13$-sets and partition $X_{2}-\left\{v_{1}, v_{2}, v_{3}\right\}$ into $c-13$-sets. This partition is equivalent to an equitable $(b+c+1,1)$-tree-coloring as required.

Finally, we obtain an equitable $(b+c)$-coloring of $K_{m, n}$ by partitioning $X_{1}$ into $b$ 3-sets and partition $X_{2}$ into $c$ 3-sets. Using Lemma 3, we have $v a_{1} \equiv\left(K_{m, n}\right)=p(b+c: m, n)$.

Lemma 8. If $m=3 b$ and $n=3 c+1$ for some positive integers $b$ and $c$, then $v a_{1} \equiv\left(K_{m, n}\right)=$ $p(b+c: m, n)$.

Proof. From Lemma 4 , the graph $K_{m, n}$ has an equitable $(q, 1)$-tree-coloring for each integer $q$ such that $q \geq b+c+2$.

Next, we show that $K_{m, n}$ has an equitable $(b+c+1)$-coloring. We initiate a partition by assigning sets $\left\{v_{1}, v_{2}\right\},\left\{v_{3}, v_{4}\right\}$ and then we partition $X_{1}$ into $b$ 3-sets, and partition $X_{2}-\left\{v_{1}, v_{2}, v_{3}, v_{4}\right\}$ into $c-13$-sets to obtain an equitable $(b+c+1)$-coloring.

Finally, we obtain an equitable $(b+c)$-coloring of $K_{m, n}$ by partitioning $X_{1}$ into $b$ 3-sets, and partition $X_{2}$ into $c-13$-sets and one 4-set. Using Lemma 3, we have $v a_{1} \equiv\left(K_{m, n}\right)=$ $p(b+c: m, n)$.

Lemma 9. If $m=3 b$ and $n=3 c+2$ for some positive integers $b$ and $c$, then $v a_{1}^{\equiv}\left(K_{m, n}\right)=$ $p(b+c+1: m, n)$.

Proof. From Lemma 4 , the graph $K_{m, n}$ has an equitable $(q, 1)$-tree-coloring for each integer $q$ such that $q \geq b+c+2$. Moreover, we can obtain an equitable $(b+c+1)$-coloring of $K_{m, n}$ by partitioning $X_{1}$ into $b 3$-sets, and partitioning $X_{2}$ into $c 3$-sets and one 2-set. Using Lemma 3, we have $v a_{1} \equiv\left(K_{m, n}\right)=p(b+c+1: m, n)$.

Lemma 10. If $m=3 b+1$ and $n=3 c+1$ for some positive integers $b$ and $c$, then $v a_{1}\left(K_{m, n}\right)=$ $p(b+c: m, n)$.

Proof. From Lemma 4 , the graph $K_{m, n}$ has an equitable $(q, 1)$-tree-coloring for each integer $q$ such that $q \geq b+c+2$.

Next, we show that $K_{m, n}$ has an equitable $(b+c+1,1)$-tree-coloring. We initiate a partition by assigning a set $\left\{u_{1}, v_{1}\right\}$ and then we partition $X_{1}-\left\{u_{1}\right\}$ into $b 3$-sets, and partition $X_{2}-\left\{v_{1}\right\}$ into $c 3$-sets to obtain an equitable $(b+c+1)$-coloring.

To obtain an equitable $(b+c)$-coloring of $K_{m, n}$, we partition $X_{1}$ into $b-13$-sets and one 4-set, and we partition $X_{2}$ into $c-13$-sets and one 4-set. Using Lemma 3, we have $v a_{1} \equiv\left(K_{m, n}\right)=p(b+c: m, n)$. 
Lemma 11. If $m=3 b+1$ and $n=3 c+2$ for some positive integers $b$ and $c$, then $v a \equiv\left(K_{m, n}\right)=$ $b+c+2$.

Proof. From Lemma 4 , the graph $K_{m, n}$ has an equitable $(q, 1)$-tree-coloring for each integer $q$ such that $q \geq b+c+2$.

It remains to show that $K_{m, n}$ has no equitable $(b+c+1,1)$-tree-coloring. Suppose to the contrary that $K_{m, n}$ has such coloring. Since $(m+n) /(b+c+1)=3$, each resulting color class has size 3 . It follows that each color class is an independent set. However, $X_{1}$ can be partitioned into at most $b 3$-sets and $X_{2}$ can be partitioned into at most $c$ 3-sets, a contradiction.

Lemma 12. If $m=3 b+2$ and $n=3 c+2$ for some positive integers $b$ and $c$, then $v a_{1}^{\equiv}\left(K_{m, n}\right)=$ $b+c+2$.

Proof. From Lemma 4, the graph $K_{m, n}$ has an equitable $(q, 1)$-tree-coloring for each integer $q$ such that $q \geq b+c+2$.

It remains to show that $K_{m, n}$ has no equitable $(b+c+1,1)$-tree-coloring. Suppose to the contrary that $K_{m, n}$ has such coloring. Since $(m+n)=3(b+c+1)+1$, one resulting color class has size 3 and the other one has size 4 . It follows that each color class is an independent set. However, either $X_{1}$ can be partitioned into at most $(b-1) 3$-sets and one 4-set and $X_{2}$ can be partitioned into at most $c 3$-sets or $X_{1}$ can be partitioned into at most $b$ 3-sets and $X_{2}$ can be partitioned into at most $(c-1) 3$-sets and one 4-set, a contradiction.

From Lemmas 5-12, we obtain the following theorem.

Theorem 2. Each $v a_{1}^{\equiv}\left(K_{m, n}\right)$ is as in Table 1.

Table 1. $v a_{1} \equiv\left(K_{m, n}\right)$ in terms of $m$ and $n$ where $b$ and $c$ are positive integers.

\begin{tabular}{|c|c|c|}
\hline$m$ & $n$ & $v a_{1}^{\equiv}\left(K_{m, n}\right)$ \\
\hline 1 & $n$ & $\lceil(n+2) / 3\rceil$ \\
\hline 2 & $n$ & $\lceil(n+3) / 3\rceil$ \\
\hline $3 b$ & $3 c$ & $p(b+c: m, n)$ \\
\hline $3 b$ & $3 c+1$ & $p(b+c: m, n)$ \\
\hline $3 b$ & $3 c+2$ & $p(b+c+1: m, n)$ \\
\hline $3 b+1$ & $3 c+1$ & $p(b+c: m, n)$ \\
\hline $3 b+1$ & $3 c+2$ & $b+c+2$ \\
\hline $3 b+2$ & $3 c+2$ & $b+c+2$ \\
\hline
\end{tabular}

\section{The Strong Equitable Vertex 1-Arboricity of $K_{k * n}$}

For $K_{k * n}$, we denote $p(q: n, \ldots, n)$ by $p(q: k * n)$.

Lemma 13. If $G=K_{k * 1}$ or $K_{k * 2}$ and $N=|V(G)|$, then $v a_{1} \equiv(G)=\lceil N / 2\rceil$.

Proof. For $q \geq\lceil N / 2\rceil$, we have $N / q \leq 2$. We obtain an equitable $(q, 1)$-tree-coloring by partitioning $V(G)$ into $q$ sets equitably. Since each set has size at most 2 , we obtain a desired coloring.

It remains to show that $G$ has no equitable $(\lceil N / 2\rceil-1,1)$-tree-coloring. Suppose to the contrary that such coloring exists. Then there is a resulting color class with size at least 3 which induces a graph with maximum degree at least 2 , a contradiction. This completes the proof.

Lemma 14. If $n=3 b$ for some positive integer $b$, then $v a_{1}^{\equiv}\left(K_{k * n}\right)=p(k b: k * n)$. 
Proof. For $q \geq 3 k b / 2$, we partition $V\left(K_{k * n}\right)$ into $q$ sets equitably. Since each set has size at most 2 , this partition leads to an equitable $(q, 1)$-tree-coloring.

For $k b+1 \leq q \leq 3 k b / 2-1$, then $2<3 k b / q<3$. It follows that there are positive integers $r$ and $s$ such that $3 k b=3 r+2 s$ and $r+s=q$. Observe that $r<k b$. Partition $X_{i}$ for each $i$ into $b 3$-sets to obtain $k b$ 3-sets. Since $r \leq k b$, we initiate a new partition by choosing $r$ sets from these $k b 3$-sets. Next, we partition the set of remaining vertices into $s$ sets equitably. Since each of 3-sets contains vertices from the same partite set, it is an independent set. Moreover, each of remaining sets has size 2 . Thus we obtain an equitable $(q, 1)$-tree-coloring for each $q \geq k b+1$.

One can easily see that $G$ has an equitable $k b$-coloring. Using Lemma 3, we have $v a_{1}\left(K_{k * n}\right)=p(k b: k * n)$.

Lemma 15. If $n=3 b+1$ for some positive integer $b$, then $v a_{1} \equiv\left(K_{2 * n}\right)=p(2 b: n, n)$ and $v a_{1} \equiv\left(K_{k * n}\right)=k b+\lceil k / 2\rceil$ for $k \geq 3$.

Proof. If $k=2$, the result comes from Lemma 10. Now assume that $k \geq 3$. For $q \geq$ $(3 k b+k) / 2$, we partition $V\left(K_{k * n}\right)$ into $q$ sets equitably. Since each set has size at most 2, this partition leads to an equitable $(q, 1)$-tree-coloring.

For $k b+\lceil k / 2\rceil \leq q \leq(3 k b+k) / 2-1$, we have $2<(3 k b+k) / q<3$. Thus there are positive integers $r$ and $s$ such that $3 k b+k=3 r+2 s$ and $r+s=q$. Consequently, $3 k b+k=3(r+s)-s=3 q-s$. Since $q \geq k b+\lceil k / 2\rceil$, it follows that $s \geq 3\lceil k / 2\rceil-k$. From $3 k b+k=3 r+2 s$, we have $r \leq k b$. Choose $X_{i}^{\prime}$ to be a $(3 b)$-subset of $X_{i}$ for each $i \in\{1, \ldots, k\}$. Partition $X_{i}^{\prime}$ for each $i$ into $b 3$-sets of size 3 . Thus we have $k b 3$-sets. Since $r \leq k b$, we initiate a new partition by choosing $r 3$-sets from these $k b 3$-sets. Next, we partition set of remaining vertices into $s$ sets equitably. Since each of 3-sets contains vertices from the same partite set, it is an independent set. Moreover, each of remaining sets has size 2. Thus we obtain an equitable $(q, 1)$-tree-coloring for each $q \geq k b+\lceil k / 2\rceil$.

It remains to show that $G$ has no equitable $(k b+\lceil k / 2\rceil-1,1)$-tree-coloring. Suppose to the contrary that $G$ has such coloring. Consider $k=4$. This yields the graph $K_{4 * n}$ has an equitable $(4 b+1,1)$-tree-coloring. But $|V(G)|=12 b+4=4+(4 b) 3$. The color classes are $4 b$-sets and one 4-set. Note that each of these color classes must be an independent set. However, there are at most $b$ color classes of size 3 to contain only vertices in $X_{i}$ where $i \in\{1,2,3,4\}$. Thus there are at most $4 b$ color classes of size 3 , a contradiction.

Consider $k=3$ or $k \geq 5$. Since $3 k b+k=3(k b-2\lceil k / 2\rceil+k+2)+2(3\lceil k / 2\rceil-k-3)$, an equitable $(k b+\lceil k / 2\rceil-1,1)$-tree-coloring yields $k b-2\lceil k / 2\rceil+k+2$ color classes of size 3 and $3\lceil k / 2\rceil-k-3$ color classes of size 2 . Note that a color class of size 3 must be independent. However, there are at most $b$ color classes of size 3 to contain only vertices in $X_{i}$ where $i \in\{1, \ldots, k\}$. Thus there are at most $k b$ color classes of size 3 , a contradiction.

Lemma 16. Let $n=3 b+2$ for some positive integer $b$. Then $v a_{1} \equiv\left(K_{k * n}\right)=k b+k$.

Proof. If $k=2$, then we obtain the result from Lemma 12. Now assume $k \geq 3$. For $q \geq(3 k b+2 k) / 2$, we partition $V(G)$ into $q$ sets equitably. Since each set has size at most 2 , the resulting partition leads to an equitable $(q, 1)$-tree-coloring.

For $k b+k \leq q \leq(3 k b+2 k) / 2-1$, we have $2<(3 k b+2 k) / q<3$. Thus there are positive integers $r$ and $s$ such that $3 k b+2 k=3 r+2 s$ and $r+s=q$. Consequently, $3 k b+2 k=3(r+s)-s=3 q-s$. Since $q \geq k b+k$, we have $s \geq k$. From $3 k b+2 k=3 r+2 s$, we have $r \leq k b$. Choose $X_{i}^{\prime}$ to be a $(3 b)$-subset of $X_{i}$ where $i \in\{1, \ldots, k\}$. Partition $X_{i}^{\prime}$ for each $i$ into $b 3$-sets to obtain $k b$ 3-sets. Since $r \leq k b$, we can initiate a new partition by choosing $r$ sets from these $k b$ sets. Next, we partition the set of the remaining vertices into $s$ 2-sets. Since each of 3-sets contains vertices from the same partite set, it is an independent set. Moreover, each of remaining sets has size 2 . Thus we obtain an equitable $(q, 1)$-tree-coloring for each $q \geq k b+k$.

It remains to show that $G$ has no equitable $(k b+k-1,1)$-tree-coloring. Suppose to the contrary that $G$ has such coloring. Since $3 k b+2 k=3(k b+2)+2(k-3)$, an equitable 
$(k b+k-1,1)$-tree-coloring has $k b+2$ color classes of size 3 and $k-3$ color classes of size 2. Note that a color class of size 3 must be independent. However, there are at most $b$ color classes of size 3 containing only vertices in $X_{i}$ where $i \in\{1, \ldots, k\}$. Thus there are at most $\mathrm{kb}$ color classes of size 3 , a contradiction.

From Lemmas 13-16, we have the following theorem.

Theorem 3. Each $v a_{1}^{\equiv}\left(K_{k * n}\right)$ is as in Table 2.

Table 2. $v a_{1} \equiv\left(K_{k * n}\right)$ in terms of $n$ where $b$ is a positive integer.

\begin{tabular}{lll}
\hline $\boldsymbol{n}$ & $\boldsymbol{v} \boldsymbol{a}_{1}^{\equiv}\left(\boldsymbol{K}_{\boldsymbol{n}, \boldsymbol{n}}\right)$ & $\boldsymbol{v} \boldsymbol{a}_{1}\left(\boldsymbol{K}_{\boldsymbol{k} * \boldsymbol{n}}\right)$ where $\boldsymbol{k} \geq \mathbf{3}$ \\
\hline 1 & 1 & $\lceil k / 2\rceil$ \\
2 & 2 & $k$ \\
$3 b$ & $p(2 b: n, n)$ & $p(k b: k * n)$ \\
$3 b+1$ & $p(2 b: n, n)$ & $k b+\lceil k / 2\rceil$ \\
$3 b+2$ & $2 b+2$ & $k b+k$ \\
\hline
\end{tabular}

\section{Conclusions}

In this paper, we extend the results of $\mathrm{Wu}, \mathrm{Zhang}$, and $\mathrm{Li} \mathrm{[10]} \mathrm{which} \mathrm{give} \mathrm{exact} \mathrm{values}$ of $v a_{1} \equiv\left(K_{n, n}\right)$ for some special cases by providing the exact values of $v a_{1}\left(K_{n, n}\right)$ for all cases. Moreover, we give a more general result by establishing exact values of $v a_{1} \equiv\left(K_{m, n}\right)$ in Theorem 2 and $v a_{1} \equiv\left(K_{k * n}\right)$ in Theorem 3 .

Author Contributions: Conceptualization, K.N. and K.M.N.; investigation, J.L.; methodology, J.L. and K.N.; validation, K.M.N. and W.R.; writing-original draft preparation, J.L.; writing-review and editing, K.N. and W.R.; supervision, K.M.N. and K.N.; funding acquisition, J.L., K.N. and W.R. All authors have read and agreed to the published version of the manuscript.

Funding: This research has received funding support from the Research Fund for Supporting Lecturer to Admit High-Potential Students to Study and Research on His Expert Program Year 2021 from the Graduate School Khon Kaen University, Thailand [Grant number 641T104-C], National Research Council of Thailand (NRCT) [Grant number N41A640141] and NSRF via the Program Management Unit for Human Resources \& Institutional Development, Research and Innovation [Grant number B05F640106].

Institutional Review Board Statement: Not applicable.

Informed Consent Statement: Not applicable.

Data Availability Statement: Not applicable.

Acknowledgments: The authors would like to thank the reviewers for their suggestions and comments. The first author was supported by the Research Fund for Supporting Lecturer to Admit High Potential Student to Study and Research on His Expert Program Year 2021 from the Graduate School Khon Kaen University, Thailand [Grant number 641T104-C]. The third author was supported by National Research Council of Thailand (NRCT) [Grant number N41A640141]. The corresponding author was supported by NSRF via the Program Management Unit for Human Resources \& Institutional Development, Research and Innovation [Grant number B05F640106]. The authors take this opportunity to thank the funders for the support.

Conflicts of Interest: The authors declare no conflict of interest.

\section{References}

1. Meyer, W. Equitable Coloring. Am. Math. Mon. 1973, 80, 920-922. [CrossRef]

2. Tucker, A. Perfect graphs and an application to optimizing municipal services. SIAM Rev. 1973, 15, 585-590. [CrossRef]

3. Irani, S.; Leung, V. Scheduling with conflicts and applications to traffic signal control. In Proceedings of the Seventh Annual ACM-SIAM Symposium on Discrete Algorithms, Atlanta, GA, USA, 28-30 January 1996; Society for Industrial and Applied Mathematics, 3600 University City Science Center: Philadelphia, PA, USA, 1996; pp. 85-94. 
4. Kitagawa, F.; Ikeda, H. An existential problem of a weight-controlled subset and its application to schedule timetable construction. Discrete Math. 1998, 72, 195-211. [CrossRef]

5. Baker, B.; Coffman, E. Mutual exclusion scheduling. Theoret. Comput. Sci. 1996, 162, 225-243. [CrossRef]

6. Blazewicz, J.; Ecker, K.; Pesch, E.; Schmidt, G.; Weglarz, J. Scheduling Computer and Manufacturing Processes; Springer: New York, NY, USA, 1998.

7. Das, S.K.; Finocchi, I.; Petreschi, R. Conflict-free star-access in parallel memory systems. J. Parallel Distri. Com. 2006, 66, 1431-1441. [CrossRef]

8. Smith, B.F.; Bjorstad, P.E.; Gropp, W.D. Domain decompoisition. In Parallel Multilevel Methods for Elliptic Partial Differential Equations; Cambridge University Press: Cambridge, UK, 1996.

9. Fan, H.; Kierstead, H.A.; Liu, G.Z.; Molla, T.; Wu, J.L.; Zhang, X. A note on relaxed equitable coloring of graphs. Inform. Process. Lett. 2011, 111, 1062-1066. [CrossRef]

10. Wu, J.-L.; Zhang, X.; Li, H.L. Equitable vertex arboricity of graphs. Discret. Math. 2013, 313, 2696-2701. [CrossRef]

11. Tao, F.Y.; Lin, W.S. On the equitable vertex arboricity of graphs. Int. J. Comp. Math. 2016, 93, 844-853. [CrossRef] 